PROCEEDINGS OF THE

AMERICAN MATHEMATICAL SOCIETY

Volume 125, Number 7, July 1997, Pages 2195-2197

S $0002-9939(97) 04097-5$

\title{
FUNCTIONS WITH PRESCRIBED QUASISYMMETRY QUOTIENTS
}

\author{
M. CHUAQUI, B. OSGOOD, AND D. STOWE
}

(Communicated by Albert Baernstein II)

Let $f$ be a continuous, increasing function of $\mathbf{R}$ into itself. Let

$$
k_{f}(x, h)=\frac{f(x+h)-f(x)}{f(x)-f(x-h)}, \quad x \in \mathbf{R}, h>0 .
$$

Observe that if $g=a f+b, a, b \in \mathbf{R}$, then $k_{g}=k_{f}$. One can show conversely that if $k_{g}=k_{f}$, then $g=f$ up to a real affine transformation. The quantity $k_{f}$ is called the quasisymmetry quotient of $f$. A function is c-quasisymmetric, $c \geq 1$, if

$$
\frac{1}{c} \leq k_{f}(x, h) \leq c .
$$

The condition (1) also implies that $f( \pm \infty)= \pm \infty$. We refer to the book by Lehto and Virtanen [1] for a thorough discussion of the role of quasisymmetric functions in the theory of quasiconformal mappings.

Due to its simple form the quasisymmetry quotient necessarily satisfies any number of algebraic identities. Two of these are sufficient, as we shall prove in the following existence theorem.

Theorem 1. Suppose that $k(x, h)$ is a positive, continuous function on the open upper half-plane $\{(x, h): x \in \mathbf{R}, h>0\}$ satisfying the identities

$$
\frac{k(x, 2 h)}{k(x, h)}=\frac{1+k(x+h, h)}{1+k(x-h, h)^{-1}}
$$

and

$$
\frac{k(x, 3 h)}{k(x, h)}=\frac{1+k(x+h, h)+k(x+h, h) k(x+2 h, h)}{1+k(x-h, h)^{-1}+k(x-h, h)^{-1} k(x-2 h, h)^{-1}} .
$$

Then there is a continuous, increasing function $f$ such that $k_{f}(x, h)=k(x, h)$.

If in addition $k(x, h)$ is bounded between $1 / c$ and $c$, then of course $f$ is $c$ quasisymmetric.

Received by the editors July 23, 1996.

1991 Mathematics Subject Classification. Primary 30C99.

(C) 1997 American Mathematical Society 
Proof. For all nonnegative integers $m$ and $n$, let

$$
\begin{gathered}
f\left(\frac{m}{2^{n}}\right)=\frac{\sum_{r=0}^{m-1} \prod_{j=1}^{r} k\left(j / 2^{n}, 1 / 2^{n}\right)}{\sum_{r=0}^{2^{n}-1} \prod_{j=1}^{r} k\left(j / 2^{n}, 1 / 2^{n}\right)}, \\
f\left(-\frac{m}{2^{n}}\right)=-\frac{\sum_{r=1}^{m} \prod_{j=0}^{r-1} k\left(-j / 2^{n}, 1 / 2^{n}\right)^{-1}}{\sum_{r=0}^{2^{n}-1} \prod_{j=1}^{r} k\left(j / 2^{n}, 1 / 2^{n}\right)} .
\end{gathered}
$$

Here we follow the usual convention that an empty sum has the value 0 and an empty product has the value 1 . Thus $f(0)=0$ and $f(1)=1$.

Equation (2) implies that $f$ is well defined. To check this it is useful to establish, by induction, the following formulas:

$$
\begin{gathered}
\prod_{j=1}^{r} k\left(j / 2^{n}, 1 / 2^{n}\right)=\frac{\prod_{j=1}^{2 r} k\left(j / 2^{n+1}, 1 / 2^{n+1}\right)+\prod_{j=1}^{2 r+1} k\left(j / 2^{n+1}, 1 / 2^{n+1}\right)}{1+k\left(1 / 2^{n+1}, 1 / 2^{n+1}\right)}, \\
\prod_{j=0}^{r-1} k\left(-j / 2^{n}, 1 / 2^{n}\right)^{-1}=\frac{\prod_{j=0}^{2 r-2} k\left(-j / 2^{n+1}, 1 / 2^{n+1}\right)^{-1}+\prod_{j=0}^{2 r-1} k\left(-j / 2^{n+1}, 1 / 2^{n+1}\right)^{-1}}{1+k\left(1 / 2^{n+1}, 1 / 2^{n+1}\right)} .
\end{gathered}
$$

Sum the first identity from $r=0$ to $m-1$ and then from $r=0$ to $2^{n}-1$. Dividing the results gives that $f\left(m / 2^{n}\right)=f\left(2 m / 2^{n+1}\right)$. Next sum the second identity from $r=1$ to $m$ and the first identity from $r=0$ to $2^{n}-1$. Dividing the results this time shows that $f\left(-m / 2^{n}\right)=f\left(-2 m / 2^{n+1}\right)$. It follows that $f$ is well defined. By construction,

$$
\frac{f\left((m+1) / 2^{n}\right)-f\left(m / 2^{n}\right)}{f\left(m / 2^{n}\right)-f\left((m-1) / 2^{n}\right)}=k\left(m / 2^{n}, 1 / 2^{n}\right), \quad m, n \in \mathbf{Z}, \quad n \geq 0 .
$$

So far, $f$ is defined at the dyadic rationals, $m / 2^{n}$, and it is strictly increasing. Let $x$ be a nondyadic number and define $f(x)$ to be the supremum of $f$ on the set of dyadic rationals less than $x$. This defines $f$ everywhere as a strictly increasing function.

Consider the set of points $(x, h)$ in the upper half-plane where $k(x, h)=k_{f}(x, h)$. By (4) this set contains all points $\left(m / 2^{n}, 1 / 2^{n}\right)$, where $m$ and $n$ are integers and $n \geq 0$. By $(2)$ it contains $(x, 2 h)$ whenever it contains $(x-h, h),(x, h)$, and $(x+h, h)$. Hence it contains all points $\left(m / 2^{n}, 2^{p}\right)$, where $m, n, p \in \mathbf{Z}, n \geq 0$. Likewise by (3) it then contains all points $\left(m / 2^{n}, 2^{p} 3^{q}\right)$, where $p, q \in \mathbf{Z}$, and $q \geq 0$. Let $\mathcal{D}$ denote the set of dyadic rationals and let $\mathcal{T}$ denote the rationals of the form $2^{p} 3^{q}, p, q \in \mathbf{Z}$, 
$q \geq 0$. Then $\mathcal{D} \times \mathcal{T}$ is dense in the upper half-plane and

$$
k(x, h)=\frac{f(x+h)-f(x)}{f(x)-f(x-h)}, \quad(x, h) \in \mathcal{D} \times \mathcal{T} .
$$

Once we know that $f$ is continuous it will then also follow that $k(x, h)=k_{f}(x, h)$ for all $x \in \mathbf{R}, h>0$.

Solving (5) for $f(x)$ yields

$$
f(x)=\frac{f(x+h)+k(x, h) f(x-h)}{1+k(x, h)}, \quad(x, h) \in \mathcal{D} \times \mathcal{T} .
$$

Letting $h$ decrease to a positive limit then implies that

$$
f(x)=\frac{f^{+}(x+h)+k(x, h) f^{-}(x-h)}{1+k(x, h)}, \quad x \in \mathcal{D}, h>0,
$$

where $f^{+}$is the right-hand limit of $f$ and $f^{-}$is the left-hand limit.

Let $x_{0} \in \mathbf{R}$. Consider $x<x_{0}$ with $x \in \mathcal{D}$ and let $x+h=x_{0}+1$. If we take the limit in (7) as $x \uparrow x_{0}$ we obtain

$$
f^{-}\left(x_{0}\right)=\frac{f^{+}\left(x_{0}+1\right)+k\left(x_{0}, 1\right) f^{-}\left(x_{0}-1\right)}{1+k\left(x_{0}, 1\right)} .
$$

On the other hand, consider $x>x_{0}$ with $x \in \mathcal{D}$ and let $x-h=x_{0}-1$. Now if we take the limit as $x \downarrow x_{0}$ we get

$$
f^{+}\left(x_{0}\right)=\frac{f^{+}\left(x_{0}+1\right)+k\left(x_{0}, 1\right) f^{-}\left(x_{0}-1\right)}{1+k\left(x_{0}, 1\right)} .
$$

Thus $f^{+}\left(x_{0}\right)=f^{-}\left(x_{0}\right)$, and $f$ is continuous at $x_{0}$. Since $x_{0}$ was arbitrary this proves that $f$ is continuous on $\mathbf{R}$, and completes the proof of the theorem.

In closing we remark that it is unlikely a single condition such as (2) on a function $k(x, h)$ will be sufficient by itself for $k$ to be a quasisymmetry quotient. For example, let $\varphi_{h}(x)=\varphi(x, h)$ be a continuous function in the upper half-plane such that $\varphi(x, 2 h)=\varphi(x, h)$ and such that the 1-parameter family of functions $x \mapsto \varphi_{h}(x)$ is uniformly quasisymmetric. If

$$
k(x, h)=\frac{\varphi(x+h, h)-\varphi(x, h)}{\varphi(x, h)-\varphi(x-h, h)},
$$

then $k$ satisfies (2), but it is not of the form $k_{f}$ unless all the functions $x \mapsto \varphi_{h}(x)$ are equivalent under the group of operations $f \mapsto a f+b$.

\section{REFERENCES}

1. O. Lehto and K. Virtanen, Quasiconformal mappings in the plane, Springer-Verlag, New York, 1973. MR 49:9202

Faculty of Mathematics, Pontificia Universidad Católica de Chile, Casilla 306, SanTiago 22, Chile

E-mail address: mchuaqui@mat.puc.cl

Department of Mathematics, Stanford University, Stanford, California 94305-2125

E-mail address: osgood@math.stanford.edu

Department of Mathematics, Idaho State University, Pocatello, Idaho 83209 